\title{
Characteristic research on lithium iron phosphate battery of power type
}

\author{
Yen-Ming Tseng ${ }^{1}$, Hsi-Shan Huang ${ }^{1}$, Li-Shan Chen ${ }^{2, *}$, and Jsung-Ta Tsai ${ }^{1}$ \\ ${ }^{1}$ College of Intelligence Robot, Fuzhou Polytechnic, No.8 Lianrong Road, Fuzhou University Town, \\ 350108, Fuzhou City, Fujian Province, China \\ ${ }^{2}$ School of Management, Fujian University of Technology, No.3 Xueyuan Road, Fuzhou University \\ Town, 350108, Fuzhou City, Fujian Province, China
}

\begin{abstract}
In this paper, it is the research topic focus on the electrical characteristics analysis of lithium phosphate iron (LiFePO4) batteries pack of power type. $\mathrm{LiFePO} 4$ battery of power type has performance advantages such as high capacity, lower toxicity and pollution, operation at high temperature environment and many cycling times in charging and discharge and so on. The charging and discharging characteristics for $\mathrm{LiFePO} 4$ batteries of power type pack have been verified and discussed by the actual experiment. Base on the 12V10AH LiFePO4 battery was proceeding on charging and discharging test with over high current value and which investigate the parameters such as the internal resistance, the related charge and discharge characteristics of $\mathrm{LiFePO} 4$ battery pack, the actual value of internal voltage and internal resistance of the battery pack and by polynomial mathmatic model to approach the accury of inner resistance on discharging mode.
\end{abstract}

\section{Introduction}

The battery is storage and energy conversion components which can be stored in the original physical energy, chemical energy or other type energy and that can be converted into electricity and released to attach circuit for application $[1,2]$. In accordance with the energy storage and release methods that can be divided into physical batteries and chemical batteries. The former are convert from the light, heat and other renewable energy to electricity and to storage in batteries or release in transmission line to loadings and consumption the power which the description type are such as solar cell [3], wind power[4], atomic force batteries and so on. Chemical batteries are chemical substances through the material of the redo reaction which by the active substances reactive effect convert from chemical energy to electricity that base on the charging and discharging apply recycle can divided into primary battery and secondary battery. Primary battery just can only discharge once and till to not drive the electrical loading so far. At the end of time in discharging mode that the battery does not chemical substances all played a chemical effect can no longer be able to provide electricity and it cannot be provided by the external power supply at after the full discharge make this battery useless.

That is because its electrochemical

Corresponding author: Sun56@ms8.hinet.net 
reaction is irreversible by the reasons of electrolyte cannot be restored by recharging step. The secondary battery is chemically converted into electrical energy and can be recharged into a battery by recharging the battery that effect is re-converted into chemical energy and it can be reused. The number of times uses will vary with the material and the application. In general, that exists in the market such as the lead-acid batteries, nickel-cadmium batteries, nickel-metal hydride batteries and lithium family batteries [5]. Different chemical type batteries rely on its working voltage, capacitance and safety of the relationship that made different applications. A lithium battery mainly refers to lithium-ion batteries. At present in the market can be found for the lithium cobalt material, lithium manganese material but the both in the use of security on the doubt there will be cause high temperature resulting in explosion or the decline in capacity of the problem. To improve the lithium batteries to lithium phosphate iron (LiFePO4) batteries [6, 7, 8] for these problems, can eliminate the user's security concerns. In this paper, the charging and discharging characteristics of power type LiFePO4 batteries pack will be by the actual experiment to verify and discussion. The study steps are following :

1) Explore and compare the dynamic characteristics of different secondary batteries of power type.

2) Discuss and decide the lithium iron phosphate (LiFePO4) battery of power type pack rated to voltage and capacity as the subject of research.

3) Discussion on the parameters and characteristics of power type $\mathrm{LiFePO} 4$ pack in charging mode and discharging mode.

4) To investigate the power type LiFePO4 pack by charging and discharging test with heavy duty.

5) Analyze the charging and discharging curves of LiFePO4 of power type with the polynomial method.

6) To find the power type LiFePO4 pack the inner resistance in charging and discharging test with heavy duty to reach the relative errors.

\section{Comparison with the LiFePO4 and the other different secondary battery}

Currently on the market mainly using the most extensive secondary battery are leadacid batteries pack, and lithium batteries such as lithium-cadmium batteries, nickel-metal hydride battery, lithium cobalt battery and LiFePO4 battery packs. Lead-acid battery because of the widely operating temperature, simple structure, technology is mature and low price characteristics to form the higher usage rate but the lower cycle life and discharge coefficient (or called Crate), higher internal resistance and high toxicity caused by high pollution shortcomings to make the replace effect by other chemical Battery packs. in this paper, it is discussed the LiFePO4 battery packs which have the advantages of high capacitance, low toxicity and no pollution, high temperature environment and good circulation performance under heavy duty charge and discharge mode, and wide sources of raw materials. Compared with other lithium family batteries packs which LiFePO4 battery packs have high efficiency energy conversion up to $95 \%$ and posses the more life cycle up to 2000 times than the other lithium family batteries packs life cycle about from 400 to 500 times. LiFePO4 battery packs is also very suitable with power supply for electric motors and for power management such as electricity scooter, pure electricity scooter and hybrid cars applications and so on, and in the future will become the mainstream of electric vehicles. 


\section{Internal resistance varying characteristics in charging and discharging mode of LiFePO4 battery pack}

In Figure 1 which $V b$ is inside voltage of battery pack and Rin is inner resistance of battery pack. Generally, battery equivalent circuit will not show Rcov and $C$. The resistance Rcov and capacitor $\mathrm{C}$ in parallel configuration and in series with the former circuit that simulation the in over-voltage states will produce the phenomenon. So that made the analogy charging process of this battery equivalent circuit diagram is more realistic and therefore improves the shortcomings of the linear model and is therefore more accurate than the linear model. And Req is the equivalent battery resistance seen by side of the voltage source, since the capacitance $C$ is the open state so that equivalent over-voltage will produce the phenomenon, and the general battery equivalent resistance Req is equal as equation (1).

$$
\operatorname{Req}=\operatorname{Rin}+\operatorname{Rcov}
$$

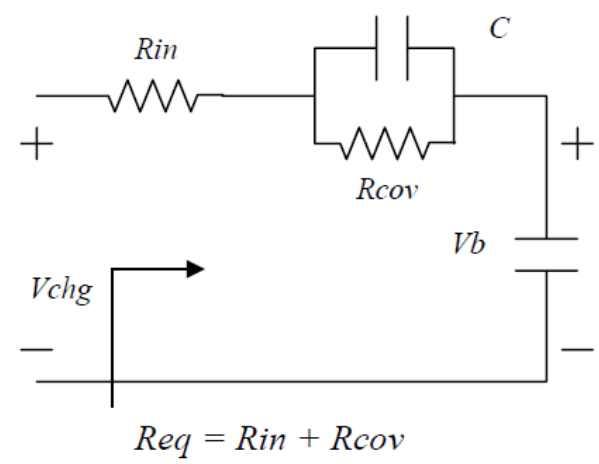

Fig. 1. Battery pack equivalent circuit diagram.

Form view of the battery pack capacity in fixed that the capacity is full or not with its battery pack inner resistance is closely related in charging mode or proceeding. In general, each battery pack capacity of the full percentage greater relative inner resistance will be made greater. In contrast, the smaller the degree of filling of each battery packs company with the smaller its internal resistance. The relationship between the external voltage and the magnitude of the battery pack resistance is by the actual circuit to test and explain.

\subsection{The relationship between with the charging voltages and currents}

Based on the specification of A123 26650 LiFePO4 battery cell as shown in the Table 1.

Table 1. LiFePO4 battery cell specification $[9,10]$.

\begin{tabular}{|c|c|}
\hline Type & A123 \\
\hline Rate voltage & $3.3 \mathrm{~V}$ \\
\hline Rate capacity & $2300 \mathrm{mAh}$ \\
\hline Weight & $73 \mathrm{~g}$ \\
\hline Charging time & $\begin{array}{l}\text { Conventional charging about 1C-2C or 3-4A and fast charging } \\
\text { about 5C or 10A. }\end{array}$ \\
\hline
\end{tabular}


A constant voltage charging circuit is designed for a $12 \mathrm{~V} 10 \mathrm{Ah} \mathrm{LiFePO} 4$ battery pack to keep the charging voltage constant and allow the charging current to be less than $3 \mathrm{C}$ which let charging current between in conventional charging and fast charging areas. The charging data is shown in Table 2. When the time is 0 for start, the initial value of the voltage is $11.48 \mathrm{~V}$ and charging current is $30 \mathrm{~A}$. When the time passes 5 seconds, the voltage rose to $11.66 \mathrm{~V}$ and then the charging current dropped to $25 \mathrm{~A}$. When the time after 50 seconds which the inside battery pack voltage rose to $12.22 \mathrm{~V}$ and then the charging current is $14 \mathrm{~A}$. According to the design of charging voltage for the battery pack, when the battery pack inside voltage reach the charging voltage will let the charging current become about $0 \mathrm{~A}$, that is called full charging. When the time passes 100 seconds, the voltage rose to $12.61 \mathrm{~V}$ and then the charging current is $11.7 \mathrm{~A}$. When the time passes 230 seconds, the voltage rose to $13.00 \mathrm{~V}$ and then the charging current dropped to $8.5 \mathrm{~A}$. And the time after 240 seconds, voltage rose to $13.00 \mathrm{~V}$ and then the charging current dropped to 3.2A we can see that when the battery slowly filling the voltage will stabilize, the current will slowly decline. Base on the data we can drown the charging curve shown in Figure 2.

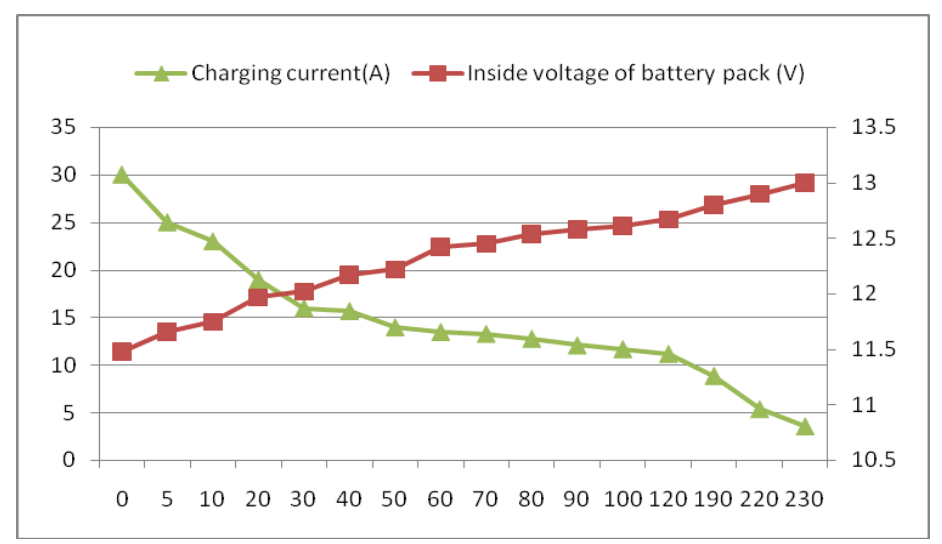

Fig. 2. LiFePO4 battery pack charging.

Table 2. LiFePO4 battery pack charging trace constant with voltage method.

\begin{tabular}{|c|c|c|}
\hline Time(second) & Inner voltage of battery pack (V) & Charging current(A) \\
\hline 0 & 11.48 & 25 \\
\hline 5 & 11.66 & 23 \\
\hline 10 & 11.75 & 19 \\
\hline 20 & 11.27 & 16 \\
\hline 30 & 12.02 & 15.7 \\
\hline 40 & 12.17 & 13.5 \\
\hline 50 & 12.22 & 13.3 \\
\hline 60 & 12.42 & \\
\hline 70 & 12.45 & 14 \\
\hline
\end{tabular}




\begin{tabular}{|l|c|c|}
\hline 80 & 12.54 & 12.8 \\
\hline 90 & 12.58 & 12.1 \\
\hline 100 & 12.61 & 11.7 \\
\hline 120 & 12.67 & 11.2 \\
\hline 190 & 12.8 & 8.9 \\
\hline 220 & 12.9 & 5.4 \\
\hline 230 & 13 & 3.6 \\
\hline 240 & 13 & 3.2 \\
\hline
\end{tabular}

\subsection{Calculation the dynamic internal voltage and equivalent inner resistance of the battery pack}

Figure 3 is a $12 \mathrm{~V} 10 \mathrm{AHLiFePO} 4$ battery pack for the power source supply and by series with a DC motor which internal resistance $R M$ is $0.07 \mathrm{ohm}$ to form a closed loop for measure the battery pack dynamic inner resistance in discharge mode under different voltage equalization. The equivalent resistance $R e q$ seen from the power supply side is shown in equation (2) and the dynamic internal resistance Rin of the battery pack is shown in equation (3). According to the discharge battery pack voltage level is divided into 13 measurements points sown in table 3 . At first time the battery pack side voltage $13.2 \mathrm{~V}$, current is $2.8 \mathrm{~A}$, supply side equivalent resistance $R e q$ is $4.71 \mathrm{ohm}$ and the battery pack internal resistance Rin is $4.64 \mathrm{ohm}$. At sixth time the battery pack side voltage $12.8 \mathrm{~V}$, current is 3.3A, supply side equivalent resistance $R e q$ is $3.88 \mathrm{ohm}$ and the battery pack internal resistance Rin is $3.81 \mathrm{ohm}$. And at the last time the battery pack side voltage $10.7 \mathrm{~V}$, current is 3.3A, supply side equivalent resistance Req is $3.24 \mathrm{ohm}$ and the battery pack internal resistance Rin is $3.17 \mathrm{ohm}$. From the table 3, it is made a result which is the higher the supply voltage with the smaller the discharging current will reach the resistance larger by loading with unchanged or fixed resistance.

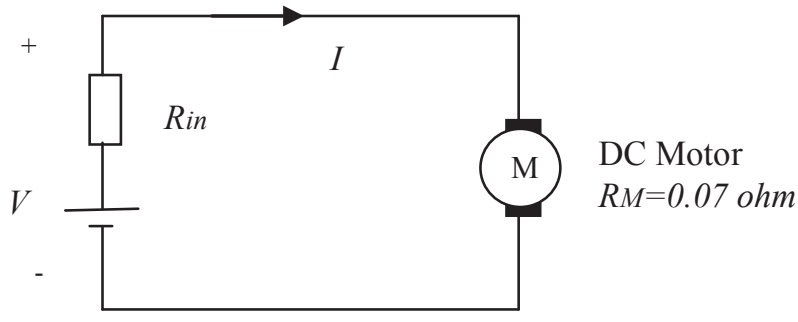

Fig. 3. LiFePO4 battery pack charging.

$$
\begin{aligned}
& R e q= V / I=R i n+R M \\
& R i n=R e q-R M
\end{aligned}
$$

With the relationship between the battery internal resistance Rin and the voltage $V$ in Table 3 that by using the third-order polynomial mathematical model of voltage $V$ is used to approach the internal dynamic intermal resistance Rin and predictive internal resistance 
value of the battery, as shown in equation (4). Using the relative error $R E v \%$ as shown in equation (5) to confirm the accuracy of the mathematical model and the Table 4 is shown in the table for each voltage class approaching the battery block internal resistance and relative error.

$$
\operatorname{Rin}=a_{0}+a_{1} \times V+a_{2} \times V^{2}+a_{3} \times V^{3}
$$

where

Rin: internal resistance of battery pack

$V$ : discharging voltage

$$
R E_{V} \%=\frac{\mid \text { Rin, actual }- \text { Rin, fit } \mid}{\text { Rin, actual }} \times 100 \%
$$

where

Rin, actual: internal resistance of battery pack which equal to Rin

Rin, fit: internal resistance approach by polynomial mathematic model.

Table 3. LiFePO4 battery pack charging trace constant voltage method.

\begin{tabular}{|c|c|c|c|c|}
\hline order Parameters & Voltage(V) & Current(A) & $\operatorname{Req}(\mathrm{ohm})$ & $\operatorname{Rin}(\mathrm{Ohm})$ \\
\hline 1 & 13.2 & 2.8 & 4.71 & 4.54 \\
\hline 2 & 13.0 & 2.7 & 4.81 & 4.74 \\
\hline 3 & 13.0 & 2.9 & 4.48 & 4.41 \\
\hline 4 & 12.9 & 3.1 & 4.16 & 4.09 \\
\hline 5 & 12.9 & 3.3 & 3.91 & 3.84 \\
\hline 6 & 12.8 & 3.3 & 3.88 & 3.81 \\
\hline 7 & 12.8 & 3.5 & 3.66 & 3.59 \\
\hline 9 & 12.8 & 3.6 & 3.56 & 3.49 \\
\hline 10 & 12.4 & 3.5 & 3.54 & 3.47 \\
\hline 11 & 11.7 & 3.4 & 3.44 & 3.37 \\
\hline 12 & 11.5 & 3.4 & 3.38 & 3.31 \\
\hline 13 & 11.1 & 3.3 & 3.36 & 3.29 \\
\hline & 10.7 & 3.3 & 3.24 & 3.17 \\
\hline
\end{tabular}

It can be seen from Table 4 that the results of the polynomial calculated by the relative error formula (5) and the measured internal resistance of the battery are within $2.21 \%$ which the maximum difference value is $0.07 \mathrm{ohm}$ and $R E_{V} \%$ is $2.21 \%$, and the minimum difference value is only $0.01 \mathrm{ohm}$ and $R E_{V} \%$ is $0.26 \%$ and the total average $R E_{V} \%$ is $1.41 \%$, so it can 
be resulted, the calculation result of formula (3) is very close to the actual value of internal resistance of battery pack. Figure 4 is relative error chart by polynomial mathematic model.

Table 4. The relative error by polynomial mathematic model.

\begin{tabular}{|c|c|c|c|c|}
\hline order & Voltage(V) & $\begin{array}{l}\text { Rin,actual } \\
\text { (ohm) }\end{array}$ & $\begin{array}{l}\text { Rin,fit } \\
\text { (ohm) }\end{array}$ & $\operatorname{Rev} \%$ \\
\hline 1 & 13.2 & 4.64 & 4.59 & $1.08 \%$ \\
\hline 2 & 13.0 & 4.26 & 4.31 & $1.17 \%$ \\
\hline 3 & 12.9 & 4.09 & 4.02 & $1.71 \%$ \\
\hline 4 & 12.8 & 3.81 & 3.80 & $0.26 \%$ \\
\hline 5 & 12.4 & 3.47 & 3.44 & $0.86 \%$ \\
\hline 6 & 11.7 & 3.37 & 3.32 & $1.48 \%$ \\
\hline 7 & 11.5 & 3.31 & 3.24 & $2.11 \%$ \\
\hline 8 & 11.1 & 3.29 & 3.23 & $1.82 \%$ \\
\hline 9 & 10.7 & 3.17 & 3.10 & $2.21 \%$ \\
\hline \multicolumn{4}{|c|}{ average } & $1.41 \%$ \\
\hline
\end{tabular}

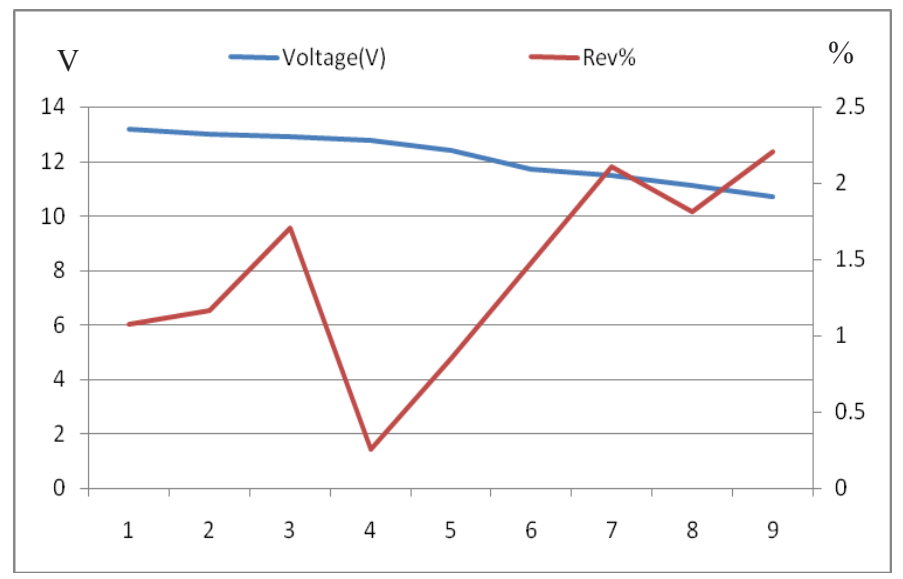

Fig. 4. The relative error chart by polynomial mathematic model.

\section{Conclusions}

For the actual circuit design to do charge and discharge test obtained the battery pack voltage, resistance and other parameters and to determine which each other dependency. And deduce the battery internal dynamic resistance and in order to other applications before the lead operations. In the charging mode, when the charging voltage is fixed that result of the battery voltage, current and internal resistance are closely related to each other. 
Similarly, in the discharge mode is also the same. In the discharge mode, the battery internal resistance and the battery potential can be approximated by the mathematical model of the third-order polynomial to the internal resistance of the battery pack and obtain its value. Through the every voltage level to statistics the total relative error is $1.41 \%$. In this article, through the relevant simple circuit can be accurate and quickly know the dynamic resistance of the battery pack and the relevant internal resistance and electricity residue of battery pack.

\section{References}

1. Le An, Dylan Dah-Chuan Lu, IEEE Transactions on Industrial Electronics, 62(2), (2015)

2. R. Rajitha, C. Balachandra Reddy, International Journal of Advanced Scientific Technologies in Engineering and Management Sciences (IJASTEMS-ISSN: 2454356X), 1(7), (2015)

3. Alejandro Pena-Bello, Meinrad Burer Martin, K. PatelDavidParra, Journal of Energy Storage, 13, (2017)

4. Sercan Teleke, Mesut E. Baran, Alex Q. Huang, Subhashish Bhattacharya and Loren Anderson, IEEE Transactions on Energy Conversion, 24(3), (2009)

5. Vinodkumar Etacheri, Rotem Marom,'Ran Elazari, Gregory Salitra and Doron Aurbach, Journal of Energy \& Environmental Science, 1(9), ( 2011)

6. Zhian Zhang, Zhiyong Zhang, Wei Chen, Guanchao Wang, Jie Li and Yanqing Lai,New Journal of Chemistry, 1(5), (2015)

7. Hu L, La Mantia F, Wu H , , Adv. Energy Mater, 1, (2011)

8. Jiajun Wang and Xueliang Sun, Energy \& Environmental Science, 8,(2015)

9. A123 system LLC , 26650 Cylindrical Cell datasheet, (2016)

10. A123 system LLC , 18650 Cylindrical Cell datasheet, (2016) 